with hemophilia. The factor concentrates used by the case patients in the US outbreak also were prepared using the S-D method of viral inactivation. Although this method inactivates enveloped viruses such as hepatitis B virus, hepatitis $\mathrm{C}$ virus, and HIV, nonenveloped viruses such as HAV and parvovirus B19 are resistant to inactivation by this method. Clotting factor concentrates manufactured by recombinant technology, which now are available, have not been shown to transmit infectious agents.

Officials of both the US Food and Drug Administration and the National Hemophilia Foundation renewed suggestions that hemophiliacs be tested for hepatitis A and that those who are susceptible be vaccinated. The CDC recommends that practitioners should consider vaccinating susceptible patients that receive clotting factor with the inactivated hepatitis A vaccine (HAVRIX R, SmithKline Beecham, Inc, Pittsburgh, PA) licensed in 1995. Additional information about this investigation of hepatitis A related to factor VIII or factor IX is available from the CDC's Hematologic Diseases Branch, and information about hepatitis A vaccine is available from CDC's Hepatitis Branch, Division of Viral and Rickettsial Diseases, NCI, telephone (404) 639-3048.

FROM: Centers for Disease Control and Prevention. Hepatitis A among persons with hemophilia who received clotting factor concentrate-United States, SeptemberDecember 1995. MMWR 1996;45(2):29-32; and Altman L. Hepatitis virus passed to hemophiliacs by clotting therapy. New York Times January 19, 1996.

\section{Staphylococcus aureus Genome Mapped}

Scientists at Human Genome Science, Inc, in Rockville, Maryland, recently reported the identification of a chemical sequence of $99 \%$ of the genome of Staphylococcus aureus. The company believes that knowledge of the full genetic sequence will assist with the development of vaccines.

One conventional method of making bacterial vaccines is to use killed bacteria to stimulate the body's immune system. The immune system then attacks major proteins on the bacterium's coat. However, these proteins often change to evade the immune attack. With the bacterium's full genome, scientists can seek out rare coat proteins that the bacterium cannot modify. This method already has been used to identify vaccine candidate proteins from Haemophilus influenzae, which was sequenced a year ago by Dr. Craig Venter and colleagues from the Institute for Genome Science, Gaithersburg, Maryland.

Dr. William Haseltine, Human Genome Science's chief executive, said the company would not release specific information about the sequence of the genome until it was patented. The sequence contains 2.8 million chemical units, coding for some 3,000 genes. Dr. Haseltine said it probably would be approximately 18 months before a patent was issued and the results of the study published.
Researchers also are developing a vaccine for toxic shock syndrome. Dr. Philippa Marrack and colleagues of the National Jewish Center for Immunology and Respiratory Medicine, Denver, Colorado, in collaboration with scientists at NeXtar Pharmaceuticals Inc, Boulder, Colorado, have prepared and tested a vaccine against the staphylococcal toxin that causes toxic shock syndrome. The researchers say that development costs for this vaccine could hit $\$ 50$ million before the drug is on the market. As a result, it may never become available to the public because of the cost.

FROM: Wade N. Company reports unlocking gene code of harmful germ. New York Times January 19, 1996, p A12.

\section{Guidelines for Xenotransplants}

An increasingly critical shortage of human donors has limited the availability and benefit of organ and tissue transplantation. This chronic shortage, combined with recent scientific and biotechnical advances, has resulted in new therapeutic approaches directed at using animal tissue in humans. Concerns have been raised about the use of xenogeneic tissues and organs for transplantation or perfusion, and the potential of both recognized zoonotic pathogens and unknown xenogeneic agents to infect individual human recipients and then spread within the human population.

Public health guidelines intended to minimize the risk for transmission of known pathogens through humanto-human transplantation do exist. Similar guidelines addressing the issue of infectious agents that may be associated with xenotransplantation are being developed by Public Health Service working groups at the CDC, FDA, and NIH. A provisional draft of these guidelines will be published in the Federal Register for public comment. Publication of a final version of these guidelines in $M M W R$ is planned for spring 1996.

In a commentary in a recent issue of Nature Medicine, Jonathan Allen, a member of the FDA panel that considered the guidelines, voiced his concerns about the risk of infectious diseases related to baboon transplants. Allan notes that baboons carry viruses that can infect humans and argues that the animals should not be used as donors for humans. Allan argued that pigs should be the only outside species used for human transplants and that federal regulations should be strict, including licensing and inspections.

FROM: Chapman LE. Guidelines on the risk for transmission of infectious agents during xenotransplants. Emerging Infectious Diseases 1995;1(4):156; and Allan J. Commentary: xenotransplantation at a crossroads: prevention versus progress Nature Medicine 1996;2(1):18.

\section{Block Grants May Weaken State Public Health Programs}

The Clinton Administration is seeking to consolidate several categorical programs into Performance Partnership Grants (PPGs). Three new PPGs would be created for CDC: for immunization; HIV/AIDS, STD, and TB; and 